\title{
A new decellularized tendon scaffold for rotator cuff tears - evaluation in rabbits
}

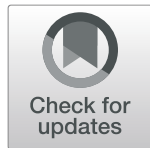

\author{
Alex de Lima Santos ${ }^{*}$ (D), Camila Gonzaga da Silva², Leticia Siqueira de Sá Barreto², Katia Ramos Moreira Leite ${ }^{3}$, \\ Marcel Jun Sugawara Tamaoki ${ }^{1}$, Lydia Massako Ferreira ${ }^{4}$, Fernando Gonçalves de Almeida ${ }^{2}$ and Flavio Faloppa ${ }^{1}$
}

\begin{abstract}
Background: Scaffolds have considerably advanced in recent years. In orthopaedic surgery, scaffolds have been used as grafts in procedures involving tendon and ligament reconstruction. This paper aimed to produce and evaluate decellularized tendon scaffolds (DTSs) from biomechanical, microscopic, macroscopic and in vivo perspectives.
\end{abstract}

Methods: Bilateral gastrocnemius muscle tendons from 18 adult New Zealand rabbits were collected. Of these 36 tendons, 11 were used as controls (Group A - control), and 25 were used in the decellularization protocol (Group B - DTS). The groups were subjected to histological, biomechanical and macroscopic analyses, and Group B - DTS was subjected to an additional in vivo evaluation. In the decellularization protocol, we used a combination of aprotinin, ethylenediamine tetraacetic acid (EDTA), sodium dodecyl sulfate (SDS) and t-octyl-phenoxypolyethoxyethanol (Triton X-100) for six days. During this period, the scaffolds were kept at room temperature on an orbital shaker with constant motion.

Results: The DTSs showed an increased cross-sectional area and inter-fascicular distance and no change in parallelism or matrix organization. The nuclear material was not organized in the DTSs as it was in the control. In the biomechanical analysis, no significant differences were found between the groups after analysing the ultimate tensile load, stiffness, and elongation at the ultimate tensile load. During the in vivo evaluation, mononuclear cell infiltration was noted.

Conclusions: The evaluated decellularization protocol generated a tendon scaffold, maintained the most important biomechanical characteristics and permitted cell infiltration.

Keywords: Scaffold, Tissue engineering, Extracellular matrix, Decellularization, Tendon

\section{Background}

Rotator cuff tears are a common cause of shoulder disability and are normally associated with weakness, loss of motion, debilitating pain and a reduced capacity for daily activities [1]. Treatment ranges from "wait-andsee" approaches to surgery. Typically, the treatment is selected according to the patient characteristics, tear size and symptoms [2, 3]. In patients with large and massive rotator cuff tears submitted for surgical treatment, the

\footnotetext{
*Correspondence: alexdels@gmail.com

'Department of Orthopedic and Traumatology, EPM - Federal University from São Paulo, 715 Napoleão de Barros, São Paulo, SP 04038-000, Brazil

Full list of author information is available at the end of the article
}

clinical results and healing process are inferior to those following surgical repairs of small lesions $[4,5]$.

An alternative by which to achieve better surgical results for massive rotator cuff tears is the use of tissue-engineered tendons, and current research has shown that scaffolds are a reliable alternative that can be used similarly to tissueengineered tendons [6, 7]. An ideal scaffold for tendon disorders and rotator cuff tears should be (a) three-dimensional in structure with high porosity; (b) devoid of cellular material to minimize the inflammatory potential, disease transmission, and host immune response; (c) cytocompatible; and (d) of sufficient biomechanical capacity to withstand rehabilitation until complete remodelling has occurred $[8,9]$. 
Scaffolds are classified as synthetic or biological. Synthetic scaffolds are generally polymers that may permit satisfactory tissue integration and facilitate cell growth. In orthopaedic practice, poly-urethane urea (SportMesh ${ }^{\oplus}$, Arthrotek, Warsaw, IN, USA), poly-L-lactide (X-repair ${ }^{\oplus}$, Synthasome, Del Mar, CA, USA) and polytetrafluoroethylene (Gore-Tex ${ }^{\oplus}$, Gore, Flagstaff, AZ, USA) have been approved by the Food and Drug Administration (FDA) and have been used in the United States of America (USA) [10, 11].

In contrast, biological scaffolds are composed of complex biological materials such as extracellular matrix (ECM) and cellular remnants [12]. Biological scaffolds are classified as follows according to the origin: autografts (the donor and recipient are the same subject), allografts (the donor and recipient are different subjects of the same species) and xenografts (the donor and recipient are subjects of different species) [13]. Autografts are most commonly used in orthopaedic surgeries; however, their limited availability is prompting studies to develop new scaffolds [13]. Allografts are an available option, but the potential chronic immune reaction and the potential risk of disease transmission are limitations of this scaffold type [14]. Finally, animal-derived xenografts have been associated with immune reactions, resulting in inflammation [14].

One approach to eliminating the risk of disease transmission or immune reactions and improving the availability of scaffolds is the decellularization process, which transforms a scaffold into a shelf product [14-16]. Decellularized tendon scaffolds (DTSs) are prepared from different protocols that include a combination of physical, chemical and enzymatic techniques $[17,18]$. Recent studies have shown reproducible results in biomechanical and histological evaluations for protocols that include combinations of chemical and enzymatic decellularization agents $[16,19,20]$.

In the literature, grafting in rotator cuff repair has been described, but the results have been mixed [21]. In a recent systematic review, lower re-tear rates and higher scores were found for patients who received rotator cuff repairs plus scaffolds [22]. Regarding the types of grafts used, the poorest results were found for xenografts, and the author did not find significant differences among autografts, allografts and synthetic scaffolds [22]. Several years ago, a randomized clinical trial was terminated due to the development of several local inflammatory reactions in patients treated with rotator cuff repairs plus xenografts (Restore Orthobiologic Implant, Depuy Orthopedics, Warsaw, Indiana) [23].

In this study, we hypothesized that a decellularization protocol with a combination of chemical agents (sodium dodecyl sulfate (SDS) plus t-octylphenoxypolyethoxyethanol (Triton X-100)) and enzymatic agents (ethylenediamine tetraacetic acid -(EDTA) plus aprotinin) would be able to produce DTSs with appropriate biomechanical characteristics that could be used in allograft transplantation. This paper aimed to produce and evaluate DTSs from biomechanical, microscopic, macroscopic and in vivo perspectives.

\section{Methods \\ Animal grouping}

Bilateral gastrocnemius muscle tendons were collected from 18 male New Zealand rabbits between 28 and 32 weeks old and weighing $3-3.5 \mathrm{~kg}$. These rabbits were provided by a farm company (Granja $R$. G., Suzano, SP, Brazil). Of these 36 tendons, 11 were used as controls (Group A - control), and 25 were used in the decellularization protocol (Group B - DTS). Among the 11 tendons used as controls, 4 were subjected to histological evaluation, and 7 were subjected to biomechanical evaluation. Of the 25 tendons subjected to the decellularization protocol, 4 underwent histological evaluation, 13 underwent biomechanical evaluation, and 8 were used for the additional in vivo analysis (Fig. 1). We used 8 (eight) extra rabbits for the in vivo analysis. The study was approved by the Institutional Review Board, and experiments were carried out according to the Animal Research: Reporting of in Vivo Experiments guidelines (ARRIVE).

\section{Gastrocnemius tendon harvest}

Tendons were collected from the rabbits following the administration of anaesthesia and euthanasia with an anaesthetic overdose (ketamine $200 \mathrm{mg} / \mathrm{kg}+$ xylazine 40 $\mathrm{mg} / \mathrm{kg}$ and tramadol $10 \mathrm{mg} / \mathrm{kg}$ ). Before anaesthesia and euthanasia, the animals were kept in individual cages under a light-dark cycle of $12 / 12 \mathrm{~h}$ with food and water available ad libitum. This study was conducted in accordance with the recommendations for the care and use of laboratory animals.

The leg was cleaned and disinfected using aseptic techniques. With an aseptic technique, a longitudinal medial posterior skin incision was made directly over the flexor digitorum superficialis [24]. The gastrocnemius tendon was carefully dissected from the flexor digitorum superficialis and soleus tendons. A complete transverse laceration was made with a surgical blade through the distal bone insertion and proximal to the muscle-tendon transition. All the tendons were removed and stored at $4{ }^{\circ} \mathrm{C}$ in phosphatebuffered saline (PBS) with $1 \%$ penicillin/streptomycin (Sigma-Aldrich, St Louis, MO, USA) for the decellularization protocol and biomechanical evaluation of the control or with $10 \%$ formaldehyde for the histological evaluation of the control.

\section{Preparation of DTSs}

Immediately after harvesting, the gastrocnemius tendons were transferred under aseptic conditions to individual tubes. Our decellularization protocol had a duration of 


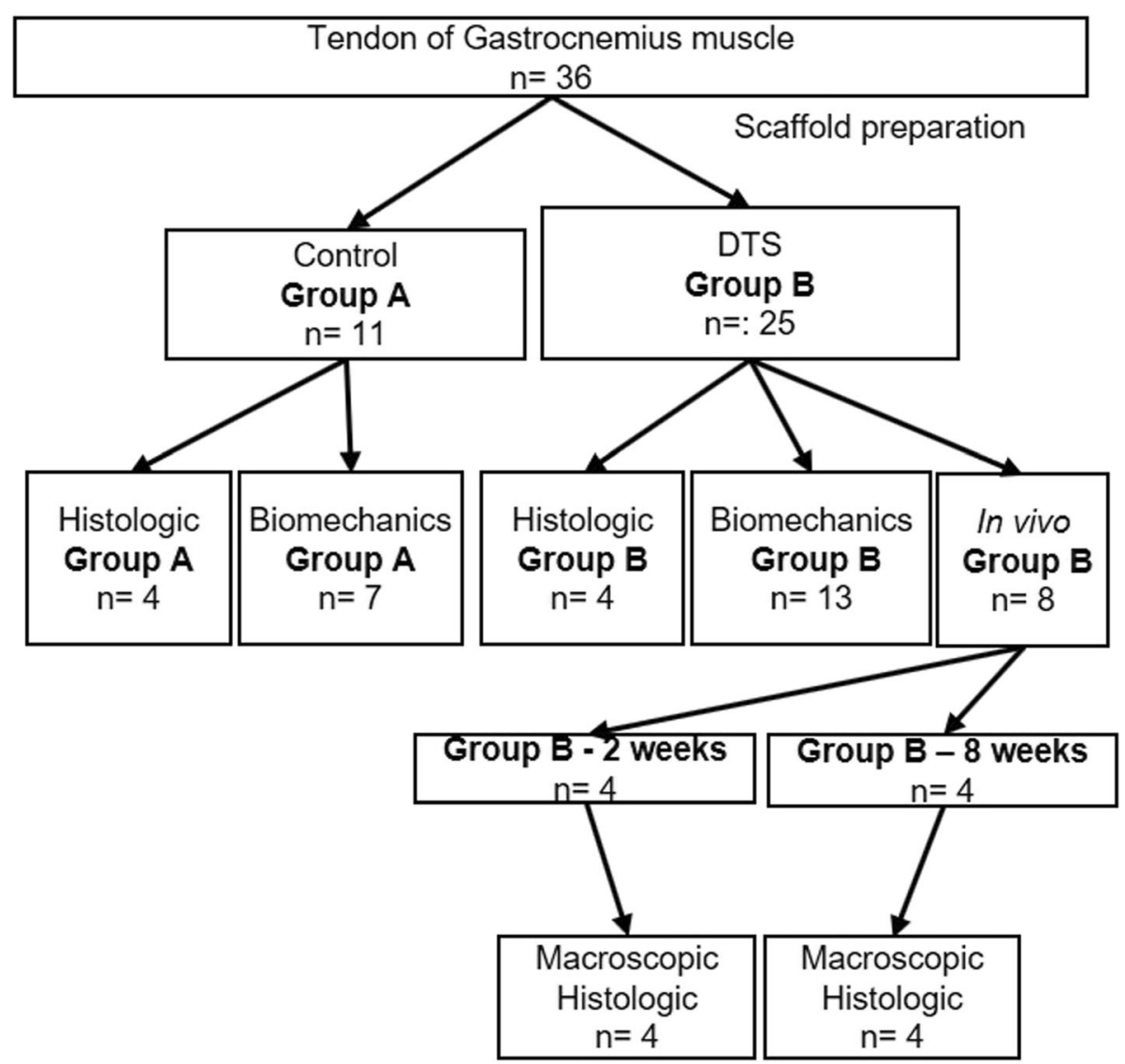

Fig. 1 Experimental flowchart detailing the processing and analysis of the control and scaffold

6 days, with daily changes of the decellularization agents under aseptic conditions. During the decellularization protocol, an individual tube was placed on an orbital shaker (MaxQ4000, Thermo Scientific, Waltham, MA, USA) at $200 \mathrm{rpm}$ and room temperature.

In the first step of the decellularization treatment, the tendons were washed with PBS for $60 \mathrm{~min}$ and then incubated in a solution containing 0.1\% EDTA (Sigma-Aldrich, St. Louis, MO, EUA) and aprotinin $10 \mathrm{~K} \mathrm{IU} / \mathrm{mg}$ (Sigma-Aldrich, St Louis, MO, EUA) for the next $24 \mathrm{~h}$ (Fig. 2 and Fig. 3).

Following the decellularization protocol, the tendons were washed with PBS for $60 \mathrm{~min}$ and then incubated with $0.5 \%$ SDS (Invitrogen, Carlsbad, CA, USA) for $24 \mathrm{~h}$. On the third day, the washing procedure was repeated with PBS, and the tendons were incubated in a solution of $1 \%$ Triton X-100 (Affymetrix, Maumme, OH, USA) for $24 \mathrm{~h}$ (Fig. 2 and Fig. 3).

On the fourth and fifth days of the protocol, the material was washed daily and maintained under constant agitation in PBS with $1 \%$ penicillin/streptomycin (Sigma-Aldrich, St Louis, MO, EUA). On the sixth day, the DTSs were placed in PBS $+1 \%$ penicillin/ streptomycin (Sigma-Aldrich, St Louis, MO, EUA) and stored at $4{ }^{\circ} \mathrm{C}$.
Tensile testing of DTSs and gastrocnemius tendons (control)

The DTSs from Group B $(n=13)$ and the controls from Group A $(n=7)$ were removed from storage, and the lengths and cross-sectional areas were measured. The cross-sectional area was measured in the middle of the scaffold or the control with a mini Vernier calliper.

The DTSs or the controls were fixed with a customized jig system consisting of metal cages with adjustable pins (Fig. 4). A specimen was mounted onto a machine for testing (AME equipment - $2 \mathrm{KN}$, Oswaldo Filizola, São Paulo, SP, Brazil) and preloaded at a predefined distance of $5 \mathrm{~mm}$ to prestretch the material. The specimen was then loaded to failure at a constant speed of $10 \mathrm{~mm} / \mathrm{min}$ until complete rupture. Immediately after preloading, the tensile load and elongation were recorded using the DynaView Standard M Software $M$ (Software DynaView Standard $M$ versão 2.7.5, Técnica Industrial Oswaldo Filizola, São Paulo, SP, Brasil) [25]. We calculated the elastic modulus, ultimate tensile stress, and stiffness using the data collected with the software and a mini Vernier Calliper [26]. 


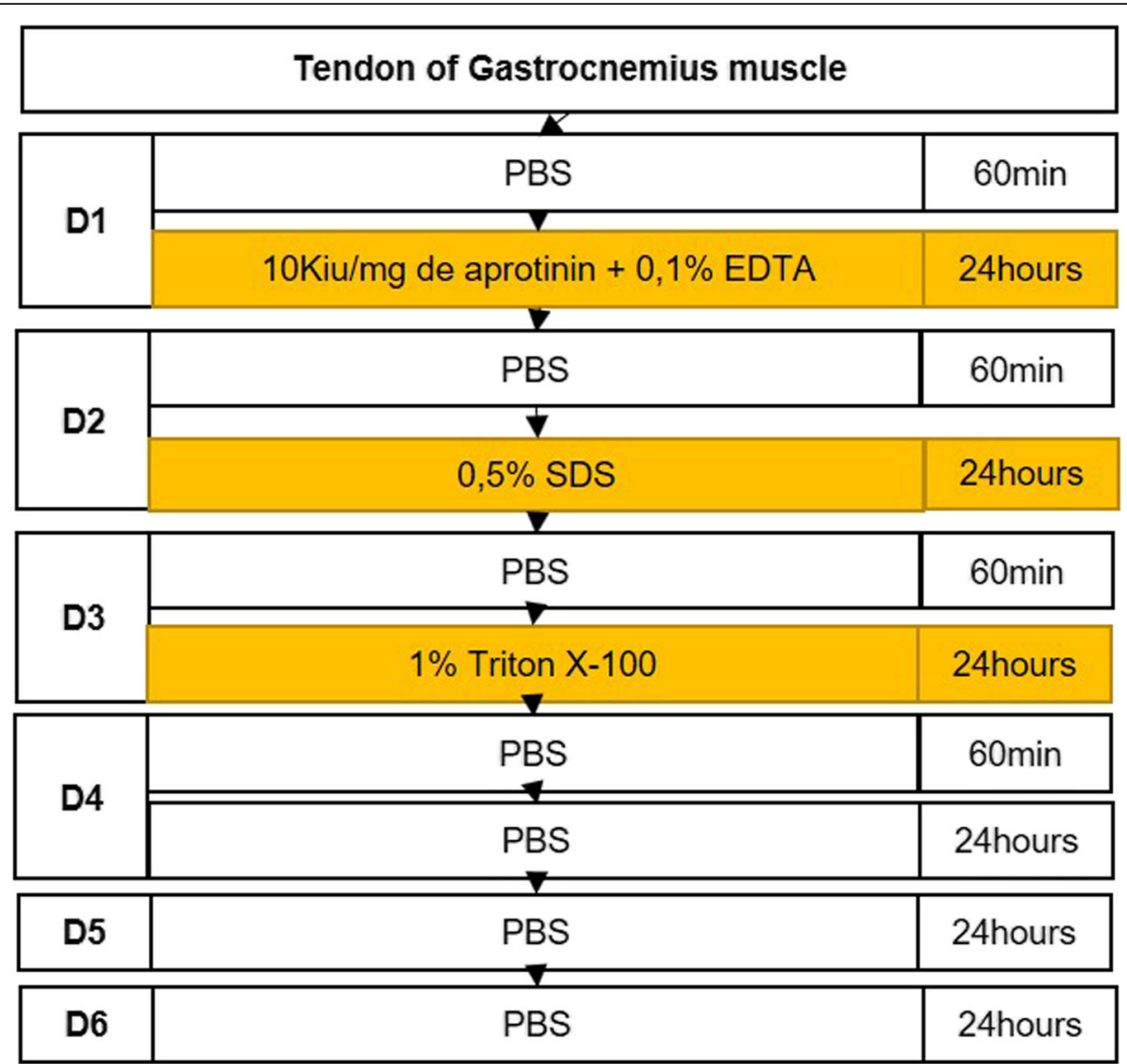

Fig. 2 Detailed protocol to produce decellularized tendon scaffolds (DTS). min: minutes, PBS: phosphate-buffered saline, SDS: Sodium Dodecyl Sulfate, Triton X-100: t-octyl-phenoxypolyethoxyethanol

\section{Histological analysis of DTSs and gastrocnemius tendons (control)}

Mid-substance portions of the specimen were placed in $10 \%$ phosphate-buffered formalin at room temperature for $6 \mathrm{~h}$. The specimens were embedded in paraffin, and a microtome was used to obtain transverse sections of $3 \mu \mathrm{m}$ thickness. The sections were mounted on slides and stained using haematoxylin and eosin ( $\mathrm{H} \& \mathrm{E})$, Masson's trichrome, 4,6-diamidino-2-phenylindole (DAPI, Invitrogen, Carlsbad, CA, EUA) and Sirius Red.

The histological sections were analysed using an Olympus IX 81/ BX51 (Olympus Corporation, Shinjuku-ku, Tokyo, Japan) fluorescence/optical microscope and a Zeiss AX10 (Zeiss, Jena, Germany) optical microscope, and images were captured with an Olympus DP71/DP72 (Olympus Corporation, Shinjuku-ku, Tokyo, Japan) or Zeiss AxioCam ICc5 (Zeiss, Jena, Germany) camera. The histological evaluation was based on the presence and organization of nuclear components, as well as the organization of the scaffold architecture $[8,20]$. Nuclear material was evaluated in a descriptive analysis using $\mathrm{H} \& \mathrm{E}$ and DAPI-stained samples. We classified nuclear basophilia removal as complete, substantial, or no removal [27]. The architectural preservation was evaluated in a descriptive analysis using H\&E, Masson's trichrome and Sirius Redstained samples [8]. We graded the architecture modification as normal, minimal disruption, or marked disruption.

\section{Macroscopic analysis of DTSs and gastrocnemius tendons (control)}

Macroscopic analysis was performed on all DTSs and controls. We analysed differences in colour, appearance, and size post-decellularization. All material collected was documented with photographs, and the results were evaluated using photos and direct visualization. The gross morphology was evaluated by two blinded participants (LSSB and CGS). Any disagreements were resolved by involving a third author (FGA).

In vivo host inflammatory response and tissue integration of DTSs in a rotator cuff model

For the in vivo evaluation, we used 8 additional male New Zealand rabbits between 28 and 32 weeks old and weighing $3-3.5 \mathrm{~kg}$. These rabbits were provided by a farm company (Granja $R$. G.). We divided the animals into two groups. Those in "Group B - 2 weeks" were euthanized 


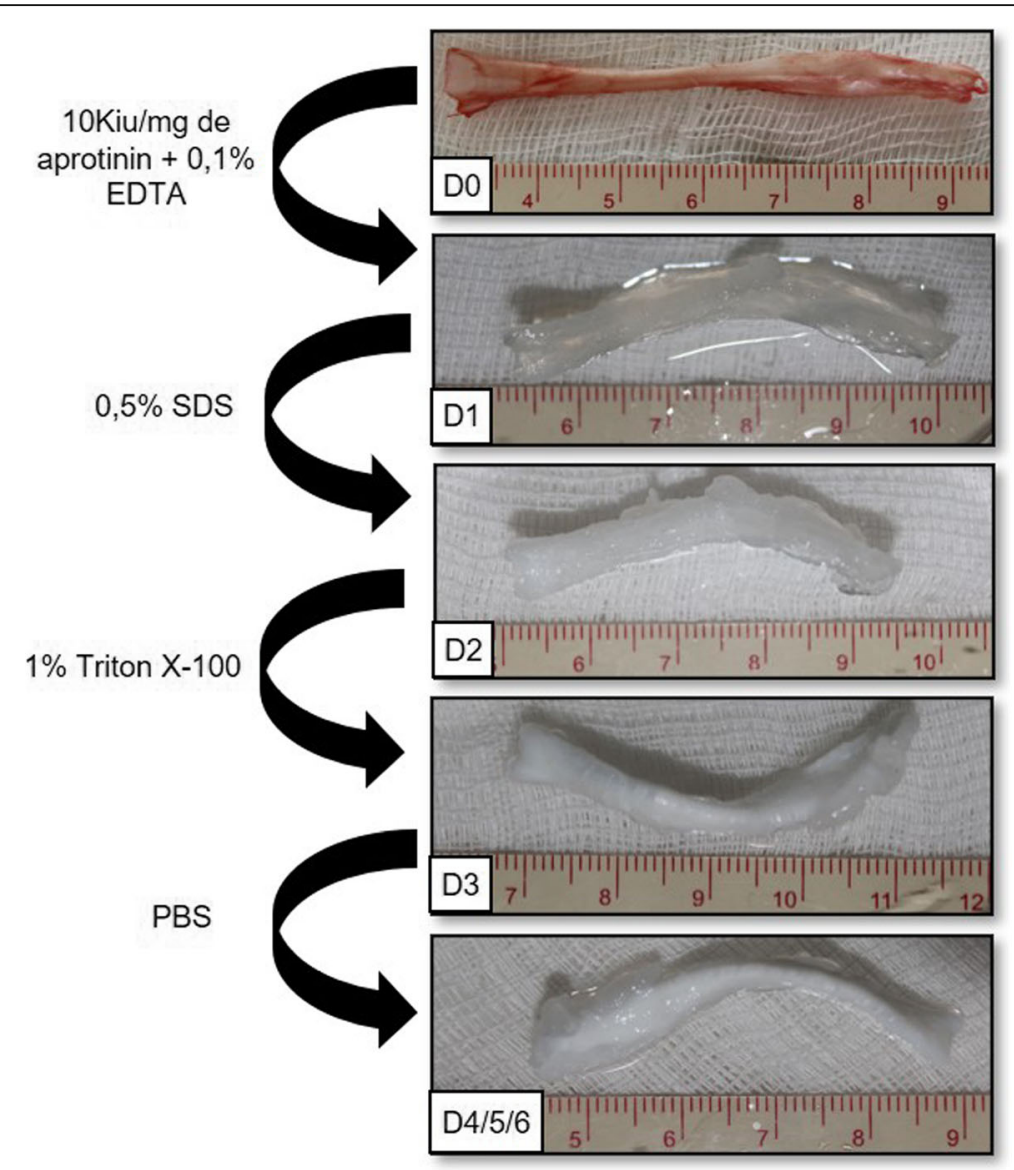

Fig. 3 Macroscopic appearance and modifications during the decellularization protocol. Macroscopic morphology has changed to white color and presented the "swelling effect"

after 2 weeks, and those in "Group B - 8 weeks" were euthanized after 8 weeks (Fig. 1). For this evaluation, we created lesions in the bilateral shoulders, but we placed a DTS only on one side, and the contralateral side served as the control. The animals were anaesthetized with a combination of ketamine $50 \mathrm{mg} / \mathrm{kg}$ + xylazine $10 \mathrm{mg} / \mathrm{kg}$ + tramadol 10 $\mathrm{mg} / \mathrm{kg}$, and a combination of tramadol $10 \mathrm{mg} / \mathrm{kg}$ and meloxicam $2-3 \mathrm{mg} / \mathrm{kg}$ was used for analgesia. The animals were kept in individual cages under a light-dark cycle of 12/12 h with food and water available ad libitum. The animals were not maintained with postoperative cast immobilization.

\section{In vivo host cell infiltration and inflammatory response to DTSs in a rotator cuff tear model}

Following the approved anaesthetic protocol, the bilateral subscapularis tendons of each animal were injured using an aseptic technique [28]. A anterolateral incision was made on the shoulder (Fig. 5-a), and the subscapularis was exposed after deltoid splitting (Fig. 5-b). A complete parallel laceration without detachment was made in the bilateral subscapularis tendons (Fig. 5-c). The laceration was not repaired, and a DTS was placed on only one side
(Fig. 6-a). The contralateral side was used as the control (Fig. 6-c). We used Nylon 4/0 (Nylon 4-0, Shalon, Alto da Boa Vista, GO, Brazil) to mark the scaffold and the lesion on the contralateral side. The animals used for the in vivo evaluation were subdivided into two groups and euthanized with an anaesthetic overdose (ketamine $200 \mathrm{mg} / \mathrm{kg}+$ xylazine $40 \mathrm{mg} / \mathrm{kg}$ and tramadol $10 \mathrm{mg} / \mathrm{kg}$ ) after two or eight weeks. The subscapularis tendon was resected from the musculotendinous junction to the bone insertion.

A histological evaluation was performed on the scaffold topography, and we evaluated the differences between specimens at two and eight weeks in terms of the cell infiltration, inflammatory response, and collagen arrangement. In the second step, we evaluated the gross morphology of the integration between the DTS and tendon and the inflammatory reaction $[7,8]$.

\section{Statistical analysis and sample size calculation}

The number of animals used for the biomechanical evaluation was determined a priori by a significance level of 0.05 and statistical power of $95 \%$ using the mean difference plus standard deviation (SD) between the ultimate tensile load 


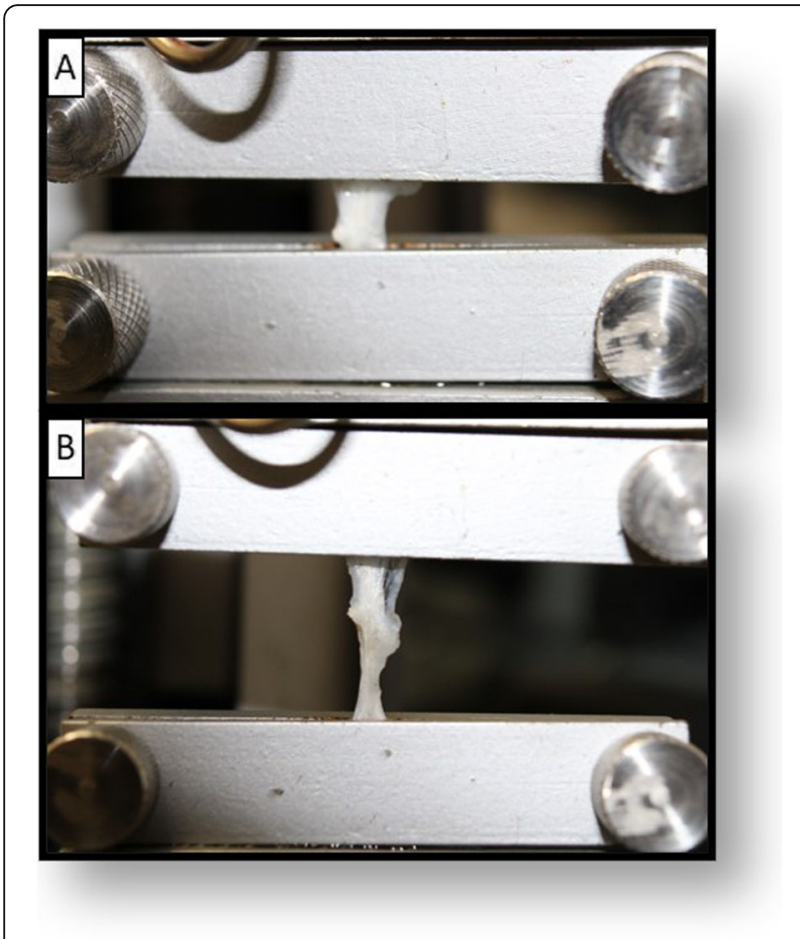

Fig. 4 Tensile testing model. a Specimen positioning in the customized jig system. $\mathbf{b}$ Tensile traction procedure showing the progressive specimen rupture of a DTS (198.24 \pm 21.05$)$ fabricated by a similar protocol and the control $(253.78 \pm 12.36)$ [20]. Using this methodology, the minimum number of samples per group was 5 (five). In a second approach, we compared the number of samples with those in relevant literature $[8,20]$.

Statistical analyses of the biomechanical data were performed by a nonparametric test (the Wilcoxon-MannWhitney test) and a power post-hoc test (Welch-Satterthwaite two sample t-test). $P \leq 0.05$ was set as the level of statistical significance. Statistical data analysis was performed using the software SAS ${ }^{\circ}$ Studio (SAS 3.8 Basic Edition; SAS Institute, Cary, NC, USA) and Excel ${ }^{\circ}$ (Microsoft $^{\circ}$ Excel $^{\circ}$ para Office 365 MSO; Santa Rosa, California, USA).

\section{Results}

\section{Tensile testing of DTSs and gastrocnemius tendon} (control)

During tensile traction, all the specimens showed a rupture in the space between the customized jig system (Fig. 4). If the specimens slipped out of the jig system, the result was discarded. In this study, we did not observe slippage during tensile testing.

The ultimate tensile load, stiffness, and elongation at the ultimate tensile load did not significantly differ

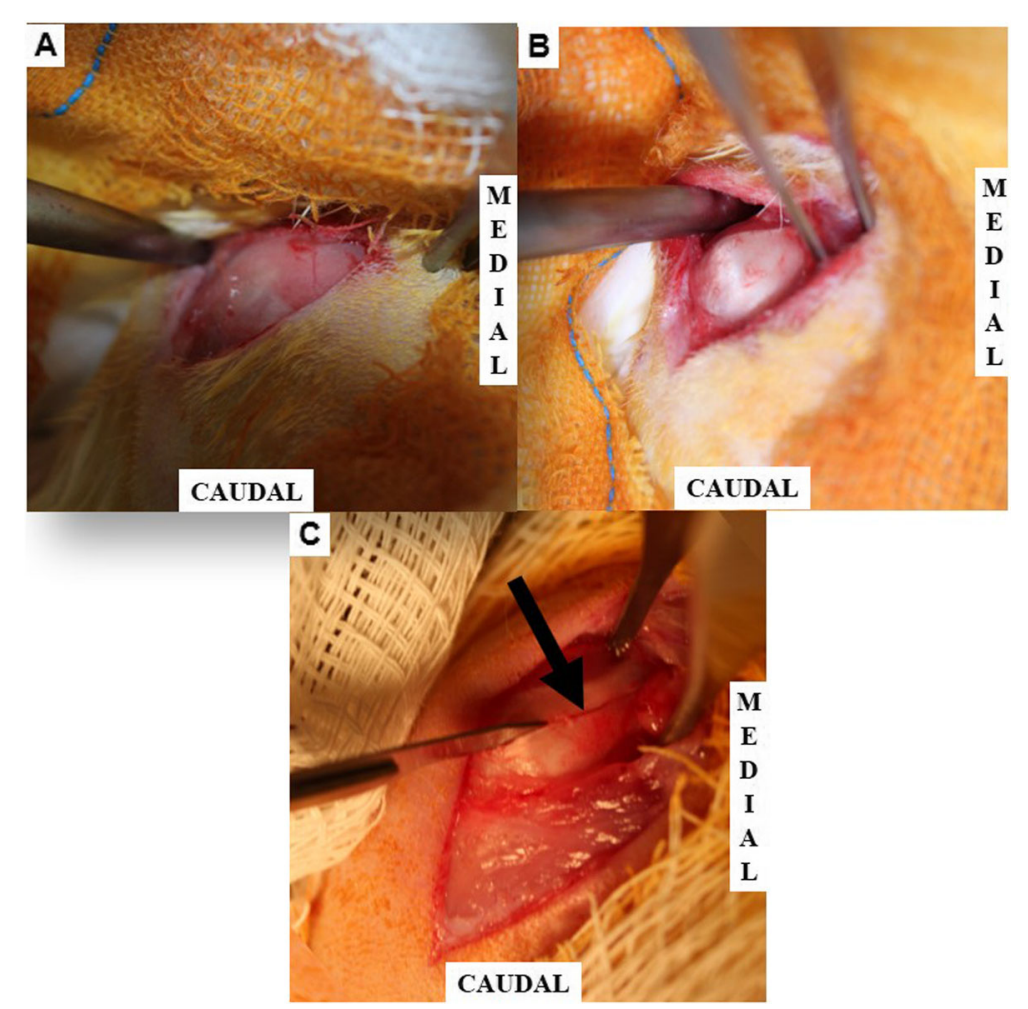

Fig. 5 The experimental model of rotator cuff lesion. a Exposition of the deltoid muscle. b Exposition of the rotator cuff (subscapularis tendon). $\mathbf{c}$ Rotator cuff lesion without detachment. Arrow: Lesion of the rotator cuff tendon 


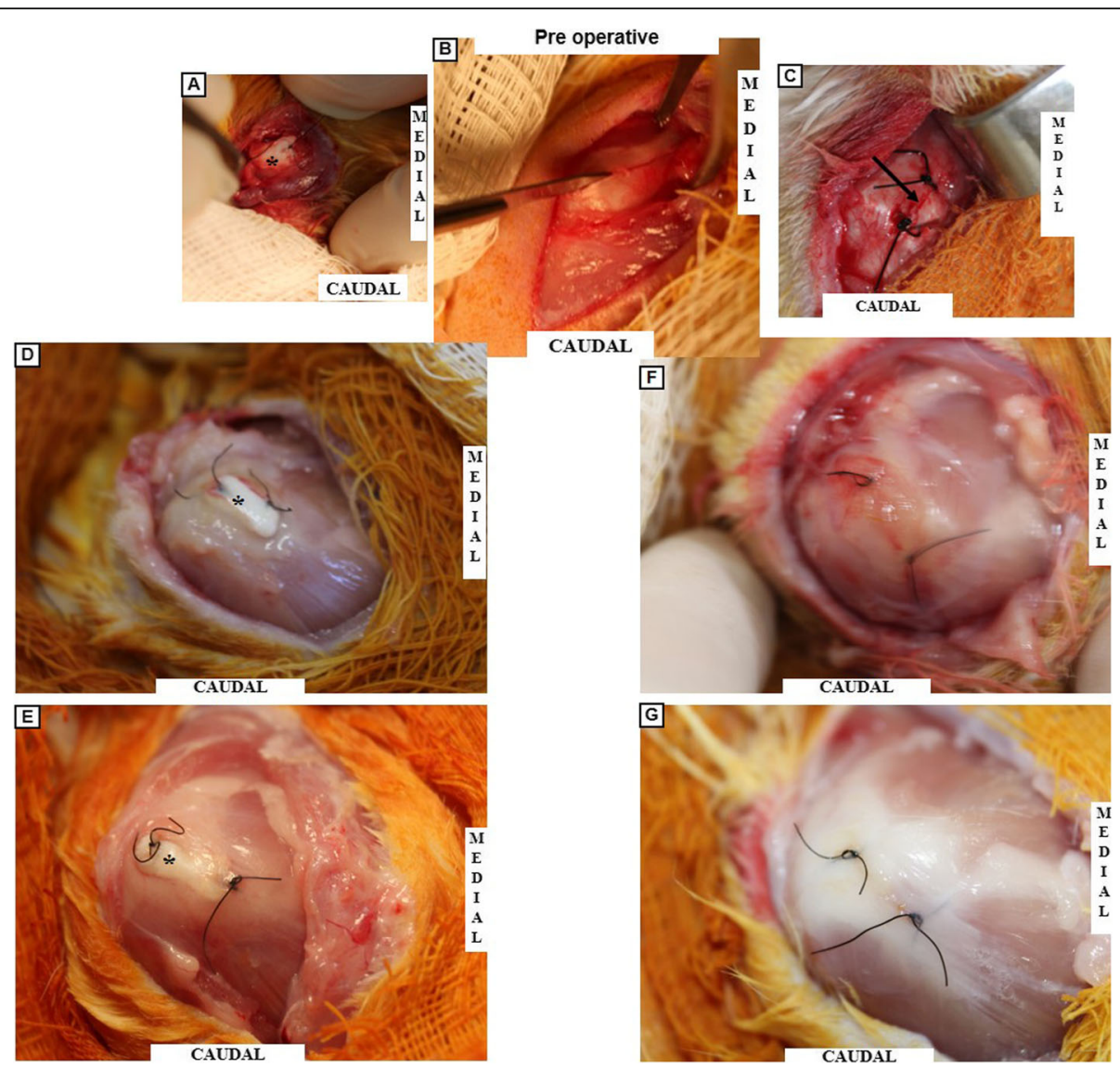

Fig. 6 Macroscopic In Vivo evaluation. a-c Pre-operative characteristic. d-e Group B - 2 and 8 weeks post-operative. f-g Control Group B 2 and 8 weeks post-operative. a Pre-operative visualization showing the DTS positioned in the rotator cuff lesion. $\mathbf{b}$ Rotator cuff lesion experimental model. c Pre-operative visualization showing the control. d 2 weeks of postoperative DTS insertion, showing inflammatory reaction and no signal of rejection. e 8 weeks post-operative appearance showing tissue integration between the DTS/tendon and a more organized tissue. $\mathbf{f}$ Control appearance after 2 weeks showing inflammatory tissue in the region of the lesion. $\mathbf{g}$ Control appearance after 8 weeks showing a cicatrized tendon. $\left(^{*}\right)$ Asterisk representing the DTS topography. Arrow: indicating the lesion region, DTS: decellularized tendon scaffold

between the groups (Table 1 and Fig. 7). The ultimate tensile load supported by the DTS was approximately $98 \%$ of that of the control. The stiffness values of the two groups were similar, and the elongation at the ultimate tensile load of the DTS was approximately $86 \%$ of that of the other group (Table 1).

The cross-sectional area, ultimate tensile stress, and elastic modulus showed statistically significant differences between the groups (Fig. 7). The crosssectional area of the DTSs increased by $164 \%$ compared to those of the controls. The elastic modulus and ultimate tensile stress of the DTSs were reduced by $61 \%$ compared to those of the control (Table 1 ). In the post-hoc analysis, the cross-sectional area and ultimate tensile stress showed power superior to $80 \%$, and the elastic modulus showed a power superior to $50 \%$. The other variables evaluated showed a power inferior to $50 \%$.

\section{Histological analysis of DTSs and gastrocnemius tendon (control)}

The controls had abundant nuclear material, as observed especially in the H\&E and DAPI staining experiments, as well as intense organization of the ECM in the H\&E, Sirius Red and Masson's trichrome staining experiments (Fig. 8).

The DTSs showed no nuclear material in H\&E staining, but DAPI staining showed the presence of nuclear material. However, in the DTSs, the nuclear material was not as organized as it was in the controls. In conclusion, we noted a substantial loss of nuclear material (Fig. 8).

We noted increases in the spaces between the collagen structures, particularly in the inter and intrafascicular spaces (Fig. 8). However, the parallelism of the fibres did not change in the DTSs, as observed in the H\&E, Masson's trichome and Sirius Red staining results (Fig. 8). In 
Table 1 Comparative table (control x DTS) relative to the biomechanical properties. No significant difference was observed in the failure load among the groups in the data relative to the ultimate tensile load, elongation at the ultimate tensile load, and stiffness. $(* p<0.05)$

\begin{tabular}{lllll}
\hline & Group I - Tendon & Group II - DTS & Scaffold / Tendon Ratio & Statistical power \\
\hline Ultimate tensile load (N), SD & $104.18(10.11)$ & $101.9(18.46)$ & $98 \%$ & 0.061 \\
Elastic modulus (MPa), SD * & $33.98(11.52)$ & $22.65(7.94)$ & $67 \%$ & 0.596 \\
Elongation at the ultimate tensile load, SD & $1.18(0.58)$ & $1.01(0.39)$ & $86 \%$ & 0.099 \\
Stiffiness (N/mm), SD & $21.87(11.45)$ & $21.99(6.34)$ & $101 \%$ & 0.053 \\
Cross-sectional-area (mm²), SD * & $3.14(0.81)$ & $5.16(1.44)$ & $164 \%$ & 0.965 \\
Ultimate tensile stress (MPa), SD * & $35.04(9.68)$ & $21.28(6.61)$ & $61 \%$ & 0.844 \\
\hline
\end{tabular}

\section{${ }^{*} p<0.05$}

Legend: $N$ Newton, SD Standard Deviation, Mpa Megapascal, $m m$ millimeter, $\mathrm{mm}^{2}$ Square millimeter, DTS Decellularized tendon scaffold

conclusion, we noted minimal disruption in all the decellularized scaffolds.

\section{Macroscopic analysis of DTSs and gastrocnemius tendons (control)}

The macroscopic analysis revealed an approximately original shape, and no pink colouration (related to eventual remaining vascularization) was observed. The collagen was well aligned, but we noted an increase in the volume of the scaffold (Fig. 3).
In vivo host cell infiltration and inflammatory response to DTSs in a rotator cuff tear model Histological analysis of in vivo host cell infiltration and inflammatory response to DTSs

We used H\&E-stained sections to evaluate the cell infiltration and inflammatory reaction. Cell infiltration of fibroblast-like host cells was observed in the DTSs, and we found more infiltration after 8 weeks than after 2 weeks (Fig. 9). Cell infiltration was concentrated in the extremities of the DTS after 2 weeks

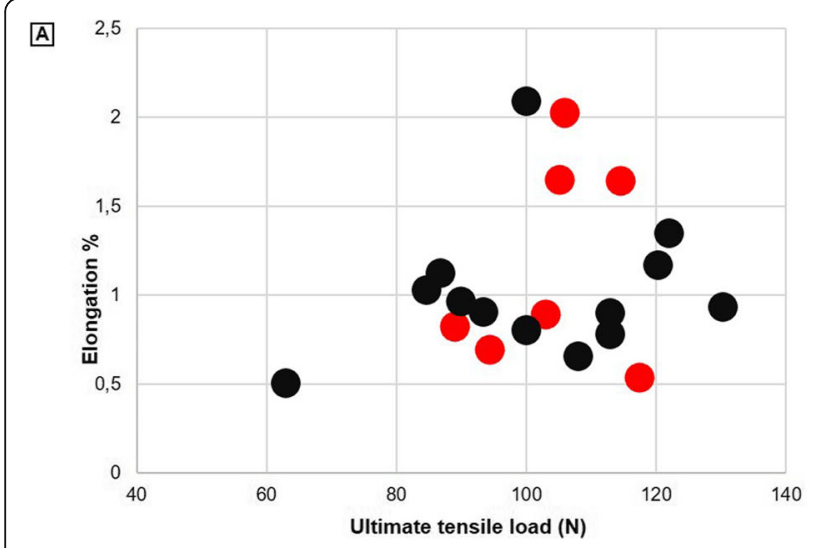

回
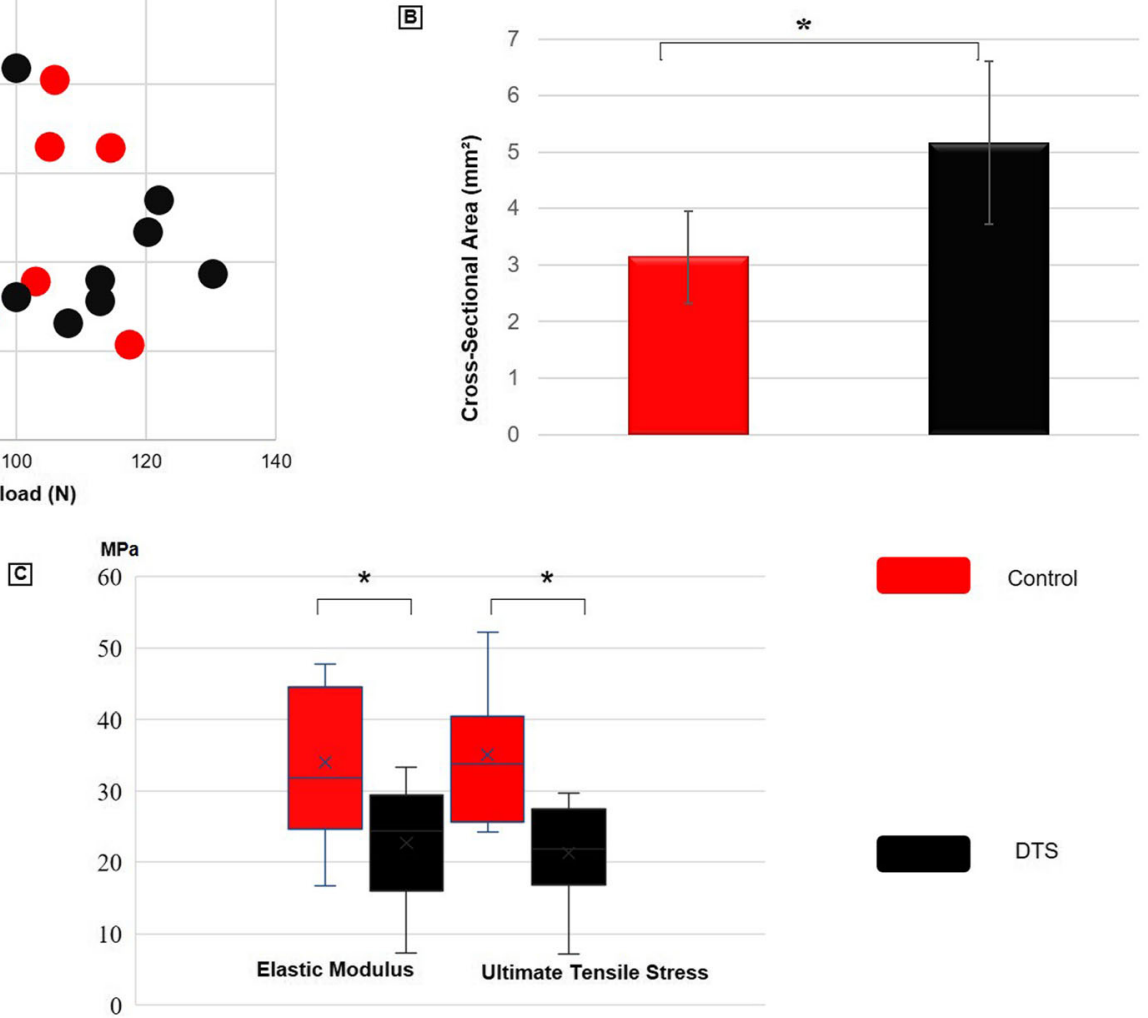

Fig. 7 Biomechanical property testing with control and DTS. a Elongation (\%) X Ultimate tensile load (N) in a dot plot presentation. b Graphic representation of mean values for cross-sectional area $\left(\mathrm{mm}^{2}\right)$. c Graphic representation of mean values for ultimate tensile strain (MPa) and elastic modulus (MPa). ( $\left.{ }^{*} p<0.05\right) . \mathrm{mm}^{2}$ : Square millimeter, MPa: Megapascal, N: Newton, DTS: decellularized tendon scaffold 


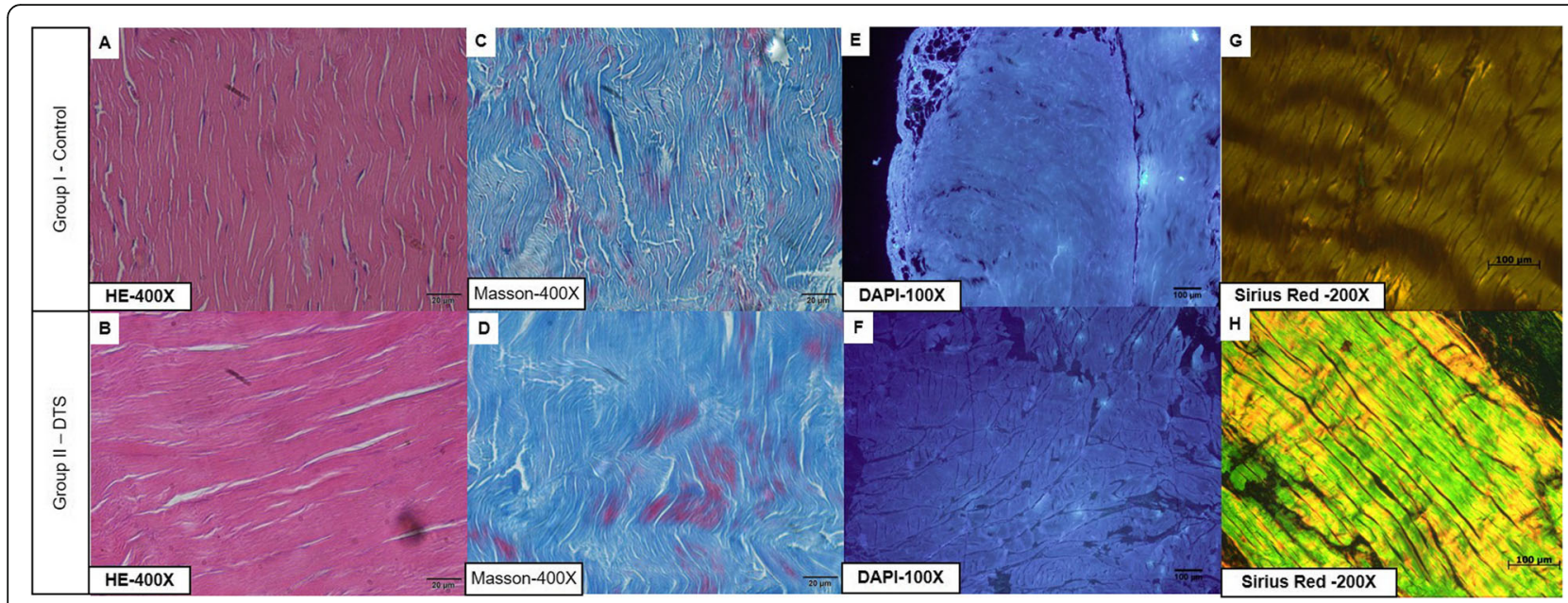

Fig. 8 Histological Analysis. a Material cellular and tendon architecture were visible. b Minimal alterations in the scaffold architecture and substantial nuclear remotion were noted. $\mathbf{c}$ Representative slides with the normal architecture of the tendon. $\mathbf{d}$ Trichome Masson slides showing minimal alteration in the architecture structure. e Punctuate nuclei (DNA) were visible in the control. $\mathbf{f}$ Only disrupted and disorganized DNA remains in the scaffold slides. $\mathbf{g}$ The normal architecture of the control. $\mathbf{h}$ Representative slides showing minimal disruption of the architecture. DTS: decellularized tendon scaffold

and disseminated in the central region after 8 weeks (Fig. 9).

In the inflammatory evaluation, we did not find significant evidence of capsule or granuloma formation, but we found some inflammatory cells, such as lymphocytes and macrophages, near the DTS, mainly after 2 weeks (Fig. 9 and Fig. 10).

\section{Macroscopic analysis of in vivo host cell infiltration and inflammatory response to DTSs}

No signs of weight loss, infection, or wound spliting were observed. In the two groups, the subscapularis insertion was in its anatomical location (Fig. 6), and the interface DTS/rotator cuff could not be detached without damaging the DTS (Fig. $6 \mathrm{~d} / \mathrm{e}$ ). We noted some

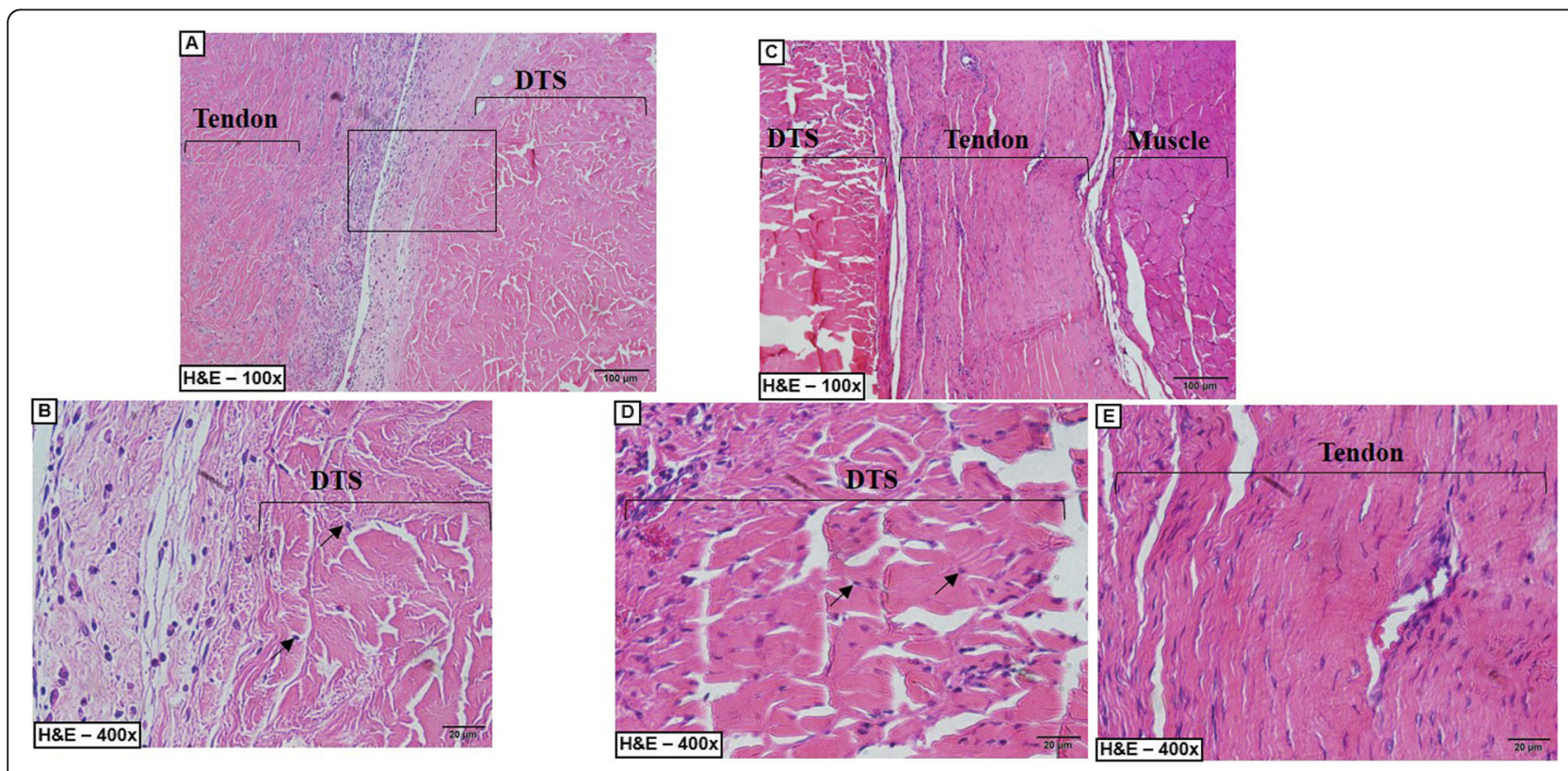

Fig. 9 Representative slides of the In vivo evaluation. a-c Group B - 2 weeks of post-operative. c-e Group B - 8 weeks of post-operative. a A large number of inflammatory cells between the tendon and the DTS. $\mathbf{b}$ Mononuclear cell infiltration (arrow) were visible in the periphery of the DTS. c Organized tissue showing the rotator cuff after the cicatrization and the integration of the DTS with the native tissue. $\mathbf{d}$ Mononuclear cell infiltration (arrow) were visible in the periphery and central region of the DTS. e Organized native tendon after 8 weeks of post-operative. DTS: decellularized Tendon Scaffold, arrow: cell infiltration 


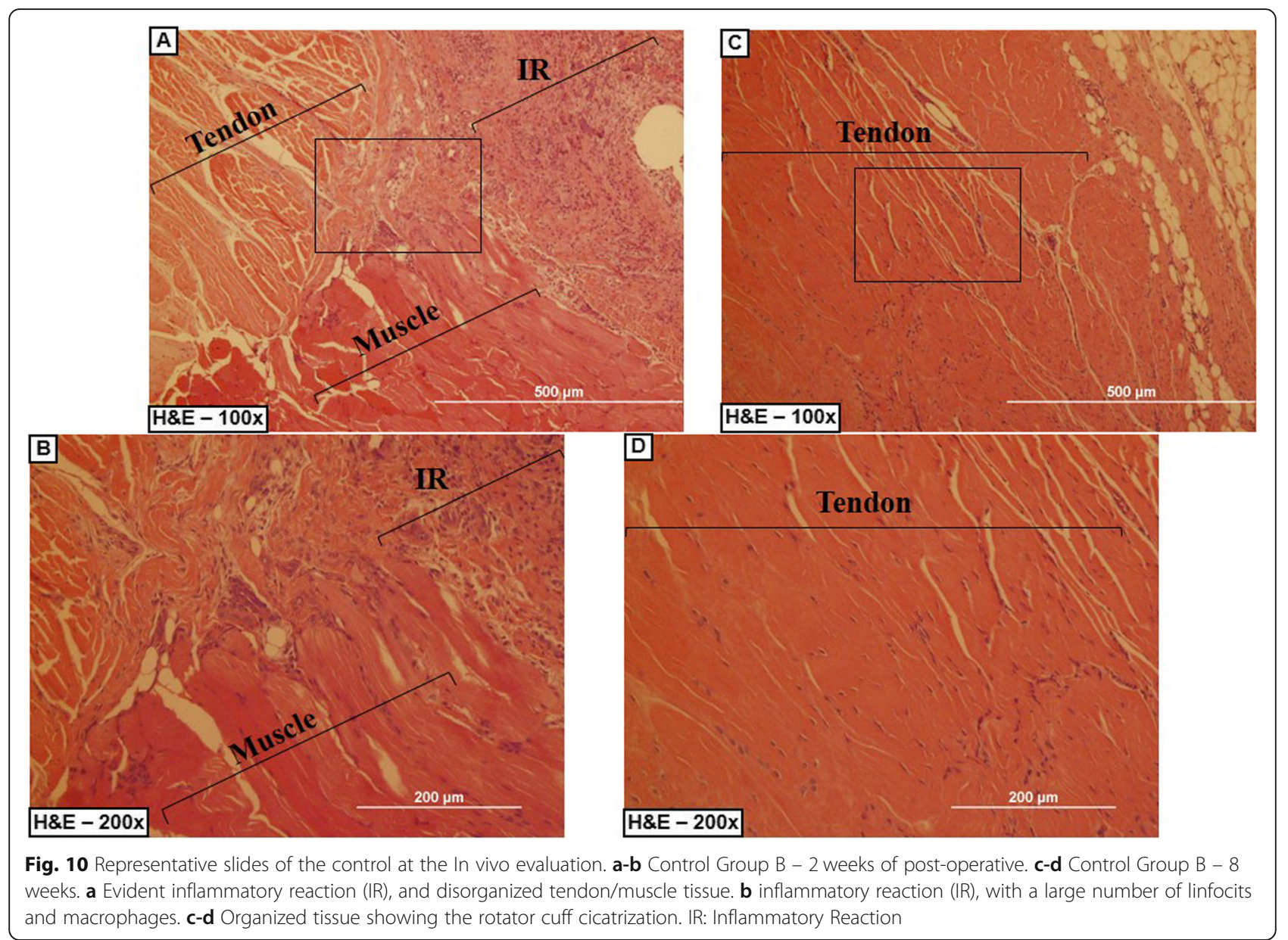

differences between the two groups. In "Group B -8 weeks", the connective tissue between the DTS and the rotator cuff was more abundant and the inflammatory reaction was less evident than in "Group B -2 weeks" (Fig. $6 \mathrm{~d} / \mathrm{e})$.

\section{Discussion}

Our most important findings were that the DTSs had a similar ultimate tensile load to that of a normal tendon, a high porosity, no organized nuclear material and showed tissue integration in the rotator cuff model. Scaffolds generated from decellularization protocols are currently used in clinical practice in various specialties $[29,30]$. In developing countries, the high cost and regulatory services limit the use of these scaffolds, unlike in developed countries. Our purpose was to fill that gap and to reproduce the promising results obtained in other studies $[8,31]$. We thus evaluated a decellularization protocol with high reproducibility using low-cost technology. We consider two options for the future. First, we could use rabbit-derived xenografts, or we could use this protocol to prepare decellularized human tendons for use in allograft transplantation. Currently, we believe that decellularized allograft transplantation is a viable option with a low likelihood of rejection.

Regarding the decellularization process, an efficient protocol should include a combination of chemical (detergents), biological (enzymatic or non-enzymatic agents) and physical agents [19, 32]. This protocol must be applied for extended periods with an appropriate technique, depending on the materials to be decellularized [17, 32]. Our protocol used a combination of two detergents (SDS + Triton X-100), a non-enzymatic agent (EDTA), a protective compound for the ECM (aprotinin), and PBS to wash the DTSs. Furthermore, the DTSs were maintained under constant agitation, following the recommendations for tendinous structures [17, 32].

The histological analysis showed an acellular scaffold, which may be related to the reduced immunogenicity and antigenicity and may maximize the host cell infiltration into the scaffold in vivo or in vitro. The biomechanical analysis showed a statistically significant increase in the cross-sectional area and decreases in the ultimate tensile stress and elastic modulus. These alterations are related to the swelling effect and did not change the minimal requirements for human tendon repair [33]. 
Based on analysing the biomechanical alterations individually, the increase in the cross-sectional area may be related to the high porosity, which may facilitate hostcell infiltration [34]. Furthermore, the elastic modulus is a direct measure of the amount of energy absorbed by the tendon, and a higher elastic modulus may be related to a stiffer and smaller deformation of the biomaterial in physiological situations [35].

Despite the biomechanical alterations related to the swelling effect, the ultimate tensile load, the elongation at the ultimate tensile load and the stiffness did not change with the protocol. In our opinion, if the ultimate tensile load and elongation are not different from those of a normal tendon, the scaffold can be used as a tendon substitute and facilitate early patient rehabilitation.

We noted some limitations. First, we did not measure the immunogenicity, toxicity, or antigenicity, and we do not know whether these biomechanical properties will persist after the use of such DTSs as a graft. The toxicity related to the decellularization process was not directly evaluated in this study, and in the clinical use of the DTS, this could be a difficulty to be solved. Second, no consensus is available on the best protocol for sterilization, which is one of the difficulties of DTSs. We attempted to use an ethylene oxide protocol as a pilot test, but the results were not satisfactory. One solution to this problem is to use peracetic acid in the protocol as an additional sterilization protocol $[8,31,36]$.

An important additional limitation is related to the a priori sample size estimate. After the post-hoc analysis, we have noted that half of the biomechanical results had a statistical power inferior to $50 \%$, and the results could be underpowered.

Some innovations related to this work should be noted. This is a new approach to using DTSs in orthopaedic procedures in a country with a large lack of soft tissue scaffolds, and we are the first to report using DTSs in a rotator cuff tear model.

In the future, we hope to start testing scaffolds in several orthopaedic procedures. DTS application in a massive rotator cuff lesion model and for anterior cruciate ligament reconstruction will be our next objectives. A different method for these studies may involve the use of mesenchymal stem cells (MSCs) as an adjuvant in the same orthopaedic procedures [25]. For these subsequent studies, the final objective will be to realise the off-theshelf availability of reliable DTSs for use in clinical practice.

\section{Conclusion}

The evaluated decellularization protocol generated a tendon scaffold, maintained the most important biomechanical characteristics and permitted cell infiltration.

\section{Abbreviations}

DAPI: 4,6-diamidino-2-phenylindole; DTS: Decellularized Tendon Scaffolds; EDTA: Ethylenediamine tetraacetic acid, 0,1\% w/v; FDA: Food and Drug Administration; H \& E: Haematoxylin and eosin; IR: Inflammatory Reaction; min: Minutes; mm: Millimetre; MPa: Megapascal; MSC: Mesenchymal stem cell; N: Newton; PBS: Phosphate-buffered saline; rpm: Rotations por minute; SD: Standard deviation; SDS: Sodium Dodecyl Sulfate; Triton X-100: T-octylphenoxypolyethoxyethanol; TnBP: 1\% tri-n-butyl phosphate; USA: United States of America

\section{Acknowledgements}

Not applicable.

\section{Ethics approval and consent participate}

This study was approved by the Ethics Committee of Animal Use of the Federal University of Sao Paulo. Protocol Number CEUA 5272080916. We obtained written informed consent to use the animals in your study from the owner of the animal.

\section{Authors' contributions}

A.L.S., conceived the project idea, performed the experiments, analyzed the data and write the manuscript. C.G.S., performed the experiments and analyzed the data. L.S.S.B., performed the experiments and analyzed the data. K.R.M.L., analyzed the data. M.J.S.T., conceived the project idea and analyzed the data. L.M.F., conceived the project idea and supervised the project. F.G.A., conceived the project idea, analyzed the data and supervised the project. F.F., analyzed the data e supervised the project. All authors discussed the results, read and approved the final manuscript.

\section{Funding}

The source of funding in this study was the Urology Research Center of the Federal University of São Paulo and the CNPQ project 311237/2018-5.

\section{Availability of data and materials}

The datasets used and/or analyzed during the current study are available from the corresponding author on reasonable request.

\section{Consent for publication}

Not applicable

\section{Competing interests}

The authors declare that have no competing interests.

\section{Author details}

'Department of Orthopedic and Traumatology, EPM - Federal University from São Paulo, 715 Napoleão de Barros, São Paulo, SP 04038-000, Brazil.

${ }^{2}$ Discipline of Urology, Department of Surgery, EPM - Federal University of Sao Paulo, São Paulo, Brazil. ${ }^{3}$ Department of Surgery, University of Sao Paulo, São Paulo, Brazil. ${ }^{4}$ Discipline of Plastic Surgery, Department of Surgery, EPM Federal University of Sao Paulo, São Paulo, Brazil.

Received: 7 February 2020 Accepted: 28 September 2020

Published online: 17 October 2020

\section{References}

1. Yamamoto A, Takagishi K, Osawa T, Yanagawa T, Nakajima D, Shitara H, et al. Prevalence and risk factors of a rotator cuff tear in the general population. J Shoulder Elb Surg. 2010;19:116-20.

2. Ejnisman B, Andreoli CV, Soares B, Peccin MS, Abdalla RJ, Faloppa F, et al. Interventions for tears of the rotator cuff in adults. Cochrane Database Syst Rev. 2009;1:CD002758.

3. Kitagaki Abechain JJ, Godinho GG, Matsunaga FT, Netto NA, Daou JP, Sugawara Tamaoki MJ. Functional outcomes of traumatic and nontraumatic rotator cuff tears after arthroscopic repair. World J Orthop. 2017;8: $631-7$.

4. Rhee YG, Cho NS, Yoo JH. Clinical outcome and repair integrity after rotator cuff repair in patients older than 70 years versus patients younger than 70 years. Arthrosc - J Arthrosc Relat Surg. 2014;30:546-54

5. Matthews TJW, Hand GC, Rees JL, Athanasou NA, Carr AJ. Pathology of the torn rotator cuff tendon: reduction in potential for repair as tear size increases. J Bone Joint Surg (Br). 2006;88-B:489-95. 
6. Ono Y, Dávalos Herrera DA, Woodmass JM, Boorman RS, Thornton GM, Lo IKY. Graft augmentation versus bridging for large to massive rotator cuff tears: a systematic review. Arthrosc - J Arthrosc Relat Surg. 2017;33:673-80.

7. Liu GM, Pan J, Zhang Y, Ning LJ, Luo JC, Huang FG, et al. Bridging repair of large rotator cuff tears using a multilayer Decellularized tendon slices graft in a rabbit model. Arthrosc - J Arthrosc Relat Surg. 2018:34:2569-78.

8. Whitlock PW, Smith TL, Poehling GG, Shilt JS, Van Dyke M. A naturally derived, cytocompatible, and architecturally optimized scaffold for tendon and ligament regeneration. Biomaterials. 2007;28:4321-9.

9. Cheng CW, Solorio LD, Alsberg E. Decellularized tissue and cell-derived extracellular matrices as scaffolds for orthopaedic tissue engineering. Biotechnol Adv. 2014;32:462-84.

10. Derwin KA, Badylak SF, Steinmann SP, lannotti JP. Extracellular matrix scaffold devices for rotator cuff repair. J Shoulder Elb Surg. 2010;19:467-76.

11. Laurencin CT, Ambrosio AMA, Borden MD, Cooper JA. Tissue engineering: orthopedic applications. Annu Rev Biomed Eng. 1999;1:19-46.

12. Sadtler K, Wolf MT, Ganguly S, Moad CA, Chung L, Majumdar S, et al. Divergent immune responses to synthetic and biological scaffolds. Biomaterials. 2019;192:405-15.

13. Andreassi A, Bilenchi R, Biagioli M, D'Aniello C. Classification and pathophysiology of skin grafts. Clin Dermatol. 2005;23:332-7.

14. Keane TJ, Badylak SF. The host response to allogeneic and xenogeneic biological scaffold materials. J Tissue Eng Regen Med. 2015;9:504-11.

15. Chong AKS, Riboh J, Smith RL, Lindsey DP, Pham HM, Chang J. Flexor tendon tissue engineering: Acellularized and reseeded tendon constructs. Plast Reconstr Surg. 2009;123:1759-66.

16. Aeberhard P, Grognuz A, Peneveyre C, McCallin S, Hirt-Burri N, Antons J, et al. Efficient decellularization of equine tendon with preserved biomechanical properties and cytocompatibility for human tendon surgery indications. Artif Organs. 2020;44:161-71.

17. Lovati $A B$, Bottagisio M, Moretti M. Decellularized and engineered tendons as biological substitutes: a critical review. Stem Cells Int. 2016;2016:1-24.

18. Su M, Zhang Q, Zhu Y, Wang S, LV J, Sun J, et al. Preparation of Decellularized Triphasic hierarchical bone-fibrocartilage-tendon composite extracellular matrix for Enthesis regeneration. Adv Healthc Mater. 2019;8:1-12.

19. Blaudez F, Ivanovski S, Hamlet S, Vaquette C. An overview of decellularisation techniques of native tissues and tissue engineered products for bone, ligament and tendon regeneration. Methods. 2020;171: 28-40.

20. Xing S, Liu C, Xu B, Chen J, Yin D, Zhang C. Effects of various decellularization methods on histological and biomechanical properties of rabbit tendons. Exp Ther Med. 2014;8:628-34.

21. Veronesi F, Borsari V, Contartese D, Xian J, Baldini N, Fini M. The clinical strategies for tendon repair with biomaterials: a review on rotator cuff and Achilles tendons. J Biomed Mater Res - Part B Appl Biomater. 2019;1:1-18.

22. Bailey JR, Kim C, Alentorn-Geli E, Kirkendall DT, Ledbetter L, Taylor DC, et al. Rotator cuff matrix augmentation and interposition: a systematic review and meta-analysis. Am J Sports Med. 2019:47:1496-506.

23. Walton JR, Bowman NK, Khatib Y, Linklater J, Murrell GAC. Restore Orthobiologic implant. J Bone Jt Surg. 2007:89:786-91.

24. Doherty GP, Koike Y, Uhthoff HK, Lecompte M, Trudel G. Comparative anatomy of rabbit and human achilles tendons with magnetic resonance and ultrasound imaging. Comp Med. 2006;56:68-74.

25. de Lima SA, da Silva CG, de Sá Barretto LS, Franciozi CE da S, Tamaoki MJS, de Almeida FG, et al. Biomechanical evaluation of tendon regeneration with adipose-derived stem cell. J Orthop Res. 2019;37:1281-6.

26. Giannini S, Buda R, Di Caprio F, Agati P, Bigi A, De Pasquale V, et al. Effects of freezing on the biomechanical and structural properties of human posterior tibial tendons. Int Orthop. 2008;32:145-51.

27. Caralt M, Uzarski JS, lacob S, Obergfell KP, Berg N, Bijonowski BM, et al. Optimization and critical evaluation of decellularization strategies to develop renal extracellular matrix scaffolds as biological templates for organ engineering and transplantation. Am J Transplant. 2015;15:64-75.

28. Grumet RC, Hadley S, Diltz MV, Lee TQ, Gupta R. Development of a new model for rotator cuff pathology: the rabbit subscapularis muscle. Acta Orthop. 2009;80:97-103.

29. He B, Zhu Q, Chai Y, Ding X, Tang J, Gu L, et al. Safety and efficacy evaluation of a human acellular nerve graft as a digital nerve scaffold: a prospective, multicentre controlled clinical trial. J Tissue Eng Regen Med. 2015;9:286-95.
30. Hu Z, Zhu J, Cao X, Chen C, Li S, Guo D, et al. Composite skin grafting with human acellular dermal matrix scaffold for treatment of diabetic foot ulcers: a randomized controlled trial. J Am Coll Surg. 2016;222:1171-9.

31. Seyler TM, Bracey DN, Plate JF, Lively MO, Mannava S, Smith TL, et al. The development of a Xenograft-derived scaffold for tendon and ligament reconstruction using a Decellularization and oxidation protocol. Arthrosc J Arthrosc Relat Surg. 2017;33:374-86.

32. Crapo PM, Gilbert TW, Badylak SF. An overview of tissue and whole organ decellularization processes. Biomaterials. 2012;32:3233-43.

33. Wang S, Wang Y, Song L, Chen J, Ma Y, Chen Y, et al. Decellularized tendon as a prospective scaffold for tendon repair. Mater Sci Eng C. 2017;77:1290-301.

34. Stabile KJ, Odom D, Smith TL, Northam C, Whitlock PW, Smith BP, et al. An Acellular, allograft-derived meniscus scaffold in an ovine model. Arthrosc J Arthrosc Relat Surg. 2010;26:936-48.

35. Suydam SM, Cortes DH, Axe MJ, Snyder-Mackler L, Buchanan TS. Semitendinosus tendon for $A C L$ reconstruction: regrowth and mechanical property recovery. Orthop J Sport Med. 2017;5:1-7.

36. Whitlock PW, Seyler TM, Parks GD, Ornelles DA, Smith TL, Van Dyke ME, et al. A novel process for optimizing musculoskeletal allograft tissue to improve safety, Ultrastructural properties, and cell infiltration. J bone Jt Surg. 2012;94:1458-67.

\section{Publisher's Note}

Springer Nature remains neutral with regard to jurisdictional claims in published maps and institutional affiliations.
Ready to submit your research? Choose BMC and benefit from:

- fast, convenient online submission

- thorough peer review by experienced researchers in your field

- rapid publication on acceptance

- support for research data, including large and complex data types

- gold Open Access which fosters wider collaboration and increased citations

- maximum visibility for your research: over $100 \mathrm{M}$ website views per year

At BMC, research is always in progress.

Learn more biomedcentral.com/submissions 